\section{Psychiatrie und Integration in den Grenzen des Sektors}

\author{
W. Werner
}

In kaum einem anderen Land wurde die Sektorpsychiatrie so eindeutig formuliert und legalisiert wie in Frankreich.

Die Berücksichtigung der dortigen Erfolge und Fehlentwicklungen erlaubt es, sie mit noch besserer Wirkung auch in anderen Ländern zu realisieren. Die mit ihr verbundenen grossen Vorzüge der raschen Verfügbarkeit, Kontinuität, Koordination aller Beteiligten und der personenzentrierten Problemnähe überwiegen die spezialistischen Möglichkeiten anderer Therapieformen, die ausserhalb des Sektorprinzips zum Tragen kämen. Mit dem sektoriellen Ansatz ist eine weitgehende Abkehr von den bisher gültigen Institutionen verbunden. Ein wesentlicher Faktor der Sektorpsychiatrie ist die Vermeidung der Desintegration in den Abläufen der Hilfe.

«Psychiatrie und Integration in den Grenzen des Sektors»: Wörter aus verschiedenen Sprachen - und dennoch, so ist darzulegen, am Ende eine allgemeinverständliche Aussage, eine jederzeit bindende Handlungsanweisung.

\section{Sektor \\ Zunächst der Sektor. Mit Sicherheit hat dieser Begriff durch die Entwicklung der französischen Psychiatrie eine besondere Bedeutung erlangt. An den Iden des März, am 15.3.1960, erging in Frankreich ein ver- bindliches Rundschreiben, das ministerielle "circu- laire du 15 mars». Es bildete die Grundlage für die Arbeit der psychiatrischen Krankenhäuser, für die Arbeit der "psychiatrie publique». Von Roger Camar, dem früheren Direktor des Landeskrankenhauses in Lorquin, stammt die Aussage: "La psychiatrie publi- que c'est la psychiatrie des pauvres». Das Prinzip des Sektorgedankens wurde in erster Linie für die Be- nachteiligten entwickelt, für die Menschen, die sich selber nicht zu helfen wissen, über die man verfügt, die man wegbringt. Heiner Kunze hat gesagt, dass die}

Korrespondenz:

Prof. Dr. med. Wolfgang Werner

SHG-Kliniken

Klinik für Psychiatrie und Psychotherapie

Trierer Strasse 148

D-66663 Merzig
Psychiatriereform vorwiegend für die Menschen gemacht wird, die keine Wahl haben. Während dieses Beitrages wird der französische Begriff der «psychiatrie publique" immer dann benutzt, wenn es um das allgemein zugängliche psychiatrische Hilfssystem geht, das von Staates wegen verbindlich installiert wurde, gleichgültig, ob es nun durch staatliche Stellen oder durch private Träger realisiert wird.

In dem ministeriellen Rundschreiben vom 15. März führte man aus, dass der psychisch Kranke so wenig wie möglich von seiner Familie und seiner Umgebung getrennt werden solle. Das entspricht auch der Erkenntnis und dem Beschluss des preussischen Landtags, der Mitte des 19. (!) Jahrhunderts im Hinblick auf die preussische Irrenanstalt in Siegburg festgestellt hatte, dass die Gemeindeferne die Heilungschancen extrem verschlechtere und dass man deshalb die zentralen Einrichtungen auf ihren sektoriellen Einzugsbereich begrenzen, die übrigen Gebiete durch zusätzliche Anstalten versorgen müsse. In Weiterentwicklung dieses Gedankens und dieses Beschlusses wurde schliesslich 1876 das Landeskrankenhaus Merzig neben vielen anderen preussischen Irrenanstalten eingerichtet. Nach einer wechselvollen Geschichte wurde es mehr als 100 Jahre später am 1.10.1998 aufgelöst und in ein Allgemeinkrankenhaus integriert. Der psychiatrische Versorgungsauftrag dieses Hauses erstreckt sich jetzt nur noch auf die sektoriellen Grenzen des Landkreises MerzigWadern. Die restlichen Landkreise oder Sektoren des Saarlandes werden von zusätzlichen, d. h. überwiegend neuen, psychiatrischen Einrichtungen versorgt [1].

Dass es im Saarland soweit kommen konnte, wie manche vielleicht mit Zustimmung, manche allerdings auch im negativen Wortverstand mit Entsetzen und Ablehnung feststellen, liegt wesentlich an der Beobachtung der französischen Entwicklung.

Dort hatte man das gemacht, was man leider oft nur bestenfalls in deutschen Anstalten vorfindet: Man hatte die jeweils bestehenden Anstalten gedrittelt, geviertelt oder gefünftelt, so dass sich dort drei, vier oder fünf Sektoren ergaben. Die Gemeinde und der Landkreis, die diese Anstalt beherbergten, hatten es gut, da sie alles, was nötig war, vor der Haustür hatten. Die anderen Kreise aber, die eben nicht die Anstalt auf ihrem Territorium hatten, lagen oft weit entfernt von ihrem Hilfssystem. So gab es z.B. bei einer der französischen Kliniken Entfernungen bis zu 120 Kilometern. Das führte zu einer Pervertierung des Sektorsystems: In das zugehörige Drittel der $120 \mathrm{~km}$ weit entfernten Anstalt kamen nur die ganz schwer gestörten Patienten, so dass dort eine Versammlung des Wahnsinns stattfand, während die am leichtesten Gestörten in der Praxis und die mässig Gestörten unter falscher Flagge auf den somatischen Stationen, in der Chirurgie, in der Inneren, in der Frauenheilkunde, behandelt wurden. Es ist für jeden Kundigen offensichtlich, was daraus hervorging. Es lohnt sich, aus dieser Vergrösserung und Vergröberung, die am Beispiel der französischen Entwicklung dargestellt wurde, auch für die Planung in kleineren Einheiten zu lernen. 
Für die Zuordnung und die Dimensionierung eines Versorgungsbereiches, eines Sektors, sind verschiedene Einflussgrössen und Faktoren zu bedenken. Die Grösse, die von dem französischen Gesundheitsministerium 1960 vorgeschlagen wurde, wird ihnen weitgehend gerecht. Man legte damals ein Gebiet mit 67000 Einwohnern zugrunde.

Die Equipe, die den Sektor versorgt (und auch ihr Chef, von dessen Engagement vieles abhängt), muss jederzeit den Überblick haben können. Das heisst, dass die Zahl der Einwohner nicht zu gross und dass die Wege nicht zu weit sein dürfen. Andererseits muss die Equipe zahlenmässig und qualitativ stark genug sein, um den unterschiedlichen Aufgaben gerecht zu werden, ob es sich nun um den ambulanten Dienst oder um eine spezielle stationäre Behandlungsaufgabe handelt, z.B. den Aufbau einer Angst- und Depressionsgruppe. Die Equipe muss ausreichend Mitarbeiter zur Verfügung haben, um während des Tages und in der Nacht zur Verfügung zu sein, z.B. durch die Gewährleistung eines ärztlichen Bereitschaftsdienstes.

Um hier zu den richtigen Werten zu kommen, muss man auf Erfahrungen aufbauen. Man kann sie aus Krankheits- oder Therapiekonzepten nicht ableiten. Es ist ähnlich wie bei der Personalverordnung zur Psychiatrie, die seinerzeit mit vielen Mühen, vielen Diskussionen, mit häufigem Ab- und Zugeben schliesslich bis zu einer einigermassen festen Form entwickelt wurde.

Nachdem in Merzig fast 10 Jahre lang konsequent Sektorpsychiatrie betrieben wurde und häufig auch durch organisatorische Änderungen Anpassungen der Sektordefinition vorzunehmen waren, kann man feststellen, dass die Grösse von 67000 sehr gut akzeptabel ist, wenn alle Leistungen vorgehalten werden sollen. Auf keinen Fall sollte sie über 100000 liegen. Wenn es organisatorisch einzurichten ist, was in Merzig einige Jahre durchführbar war, die Gegend weiter zu subsektorisieren, dann ergeben sich noch bessere Bedingungen: Bei Stationen für jeweils 33000 Einwohner und übergreifenden Diensten für insgesamt 100000 Einwohner kam es zu den besten Ergebnissen. Die Grösse des in Merzig empirisch als ideal ermittelten Subsektors entspricht genau den Empfehlungen, die auch Mosher in seiner Gemeindepsychiatrie empfohlen hat [2].

Nach diesen Angaben zur Einwohnerzahl des Sektors und zur Kapazität des Versorgungssystems sollen noch einige prinzipielle Faktoren der Sektorversorgung genannt werden. Das Wichtigste ist natürlich die Verhinderung der Desintegration (s. später). Damit hängt eng die schnelle Verfügbarkeit der Dienste zusammen, also die räumliche Nähe. Für Menschen, die längerfristige Störungen entwickeln - und darum handelt es sich ja meistens bei den schwerer kranken psychiatrischen Patienten - wird durch die räumliche Nähe mit der damit verbundenen Verfügbarkeit und wird durch die klare Definition der verantwortlichen professionellen Equipe die kontinuierliche Betreuung gewährleistet: kontinuierlich in den Personen, die sie ausüben, kontinuierlich deshalb aber auch in den Konzepten und Zielen, die zugrunde liegen und angestrebt werden.

Natürlich ist der Sektor keine rein französische Erfindung. Ähnliche Gedanken liegen auch den britischen, den italienischen und den deutschen, sicher auch den schweizerischen Entwicklungen zugrunde. Der Geist dieses psychiatrischen Zuganges weht schon durch den Enquête-Bericht und verstärkt sich in dem Papier der Expertenkommission, das ausdrücklich die chronisch Kranken an die erste Stelle rückt, also die "psychiatrie des pauvres» [3, 4]. Auch finden sich in dem deutschen Expertenpapier viele praktische Hinweise, die die Realisierung dieses Geistes ermöglichen sollen, ob es sich nun um den gemeindepsychiatrischen Verbund, um die Tagesstätten oder um die Institutsambulanz handelt.

Trotzdem drängt sich immer wieder der Eindruck auf, dass man zu vergessen droht, um was es eigentlich geht, nämlich um die Verantwortung der "Familie für ihre Kinder»: die Verantwortung des kommunalen Kreises für seine Bürger, um die Suche nach Möglichkeiten, den zur Zeit Gestörten an seinem sozialen Platz zu halten, um die Nutzung von bereits vorhandenen Unterstützungsmöglichkeiten oder um die Entwicklung von alternativen Hilfen. Es geht in erster Linie um den einzelnen in der Gemeinschaft, und es geht nicht darum, dass Psychiatrie in ihren verschiedenen Variationen verwirklicht wird. Wenn man sie an der einen oder anderen Stelle brauchen kann, um den sozialen Platz des psychisch Gestörten zu sichern, dann ist es gut.

\section{Integration}

Auch der zweite Begriff kommt aus dem romanischen Sprachbereich: Integration.

Dabei geht es nicht um die Ausländer oder um die Sektierer; es geht um die psychisch Gestörten und damit um den Gegenstand des dritten Abschnitts, um die Psychiatrie. Integration und Psychiatrie müssen zumindest stellenweise zusammen besprochen werden, auch wenn man zuweilen den Eindruck hat, als ob man gleichzeitig von Feuer und Wasser redete. Beide sind Gegensätze - und trotzdem braucht man, aufeinander bezogen, hin und wieder das eine und das andere, das Wasser, um das Feuer zu löschen, das Feuer, um das Wasser zum Sieden zu bringen.

Die bisherigen psychiatrischen Institutionen waren im wesentlichen dazu gemacht, die Menschen wieder an ihren sozialen Platz zurückzuführen, waren oftmals dazu bestimmt, die Folgen des psychiatrischen Systems zu beheben. So gab es z. B. in Deutschland innerhalb vieler Anstalten spezielle Enthospitalisierungsstationen. Der Aufenthalt an den zur Hilfe bestimmten Orten hatte sehr oft zum Verlust der wichtigen Lebensfelder geführt: der Wohnung, der Arbeit, des Freundeskreises.

Es ist eine prinzipielle Gefahr aller institutionellen Hilfen, dass sie den einzelnen Menschen zu einer speziellen Gruppe hinzudefinieren, in ein spezielles Ghetto einweisen, z. B. in eine Rehabilitationseinrich- 
tung für psychisch Kranke, dass sie ihn also desintegrieren. Aufgabe der Psychiatrie muss es aber sein, die wesentlichen Ziele ohne diese Nebenwirkungen $\mathrm{zu}$ erreichen. Von wenigen Ausnahmen abgesehen, besteht die Aufgabe psychiatrischen Handels im wesentlichen darin, die Desintegration zu vermeiden. Das bedeutet, dass das Hilfssystem nicht zum Verlassen oder zum Verlust des sozialen Platzes führen darf, dass es nicht stigmatisiert. Das bedeutet, dass es so wenig wie möglich als Institution erkennbar ist.

Ein Hilfssystem, das nach diesen Gesichtspunkten arbeitet, kann aber nur kleinräumig aktiv sein, um jederzeit zu erfassen, wo es brennt, oder um jederzeit gerufen werden zu können und auch um allen anfallenden Aufgaben gerecht zu werden. Das heisst, es muss nicht nur nahe sein, die Gruppe, für die es Verantwortung trägt, darf auch nicht zu gross sein.

Innerhalb des Kreises Merzig-Wadern bemüht man sich sehr darum, die psychiatrische Hilfe nur auf den psychiatrischen Teil zu begrenzen. Der örtliche Hilfsverein, der den Namen TRIAS trägt, ist voll verantwortlich innerhalb der "psychiatrie des pauvres" für die Bedürfnisse des Wohnens, der Arbeit und der Tagesgestaltung. Er wird weder klinisch kontrolliert noch koordiniert. Von der Tagesstätte abgesehen, die natürlich auch ein kleines Ghetto ist, das manchmal von wohlmeinenden Mitbürgern besucht wird, benutzt der Hilfsverein die normalen Strukturen: Die betreuten Wohnungen liegen in normalen Häusern, so dass die übrigen Leute in der Strasse gar nicht wissen, wen sie in ihrer Nachbarschaft haben. Die Arbeit findet in normalen Betrieben statt, je nach Behinderungsgrad. Es ist nicht zu fassen, in welchem Umfang hier Hilfsmöglichkeiten gefunden werden, wenn man nur die Räume klein genug macht und wenn sich jemand mit aller Kraft darum bemüht. Von den $54 \mathrm{~Pa}$ tienten, die sich in den letzten 4 Jahren gemeldet haben oder die angesprochen wurden, fanden 38 eine Arbeit in Betrieben ihrer Beschäftigungswahl. Knapp 20\% dieser Patienten erhielten anschliessend ein normales Arbeitsverhältnis mit sozialer Absicherung.

\section{Psychiatrie}

Vom romanischen Sprachraum geht es jetzt in den griechischen, es geht zur Psychiatrie - und dabei muss das Gegenteil herauskommen von der griechischen Insel Leros. Es wurde bereits ausgeführt, was am wichtigsten ist: Es geht um die Verteidigung und um den Erhalt des sozialen Platzes. Darüber hinaus geht es natürlich noch um andere Aufgaben, die aber für die Diskussion und Planung der öffentlichen Psychiatrie eine mehr randständige Bedeutung haben. Trotzdem werden sie sehr oft als hauptsächliches Gegenargument ins Feld geführt. Das ist aber auch etwas spezifisch Psychiatrisches, dass nämlich die Ausnahme zur Regel gemacht wird, wie man sehr schön am Beispiel der geschlossenen Stationen zeigen kann.

Betrachtet man die Einwirkungsmöglichkeiten der Psychiatrie der Reihe nach, so gibt es Dinge, die man nur im Krankenhaus machen kann, z.B. eine stereotaktische Operation. Die braucht man heute ja nicht mehr, wohl aber eine Infusionsbehandlung und intensive Überwachung bei einer bakteriellen Meningoenzephalitis, die zu einer körperlich bedingten Verrücktheit geführt hat. Die extrem schweren Depressionen wird man wohl ebenfalls in Zukunft stationär behandeln, wenn man die Menschen nicht unnötig leiden lassen will. Diese und ähnliche Aufgaben kann man aber in der ganz überwiegenden Zahl der Fälle in dem vor Ort vorhandenen psychiatrischen Krankenhaus durchführen, wenn die einzelnen Ärzte, was man verlangen sollte, auf dem neuesten Stand sind, und das, was sie nicht wissen, nachlesen oder telefonisch erfragen. Hochspezialisierte, nur in einzelnen Zentren mögliche Behandlungen, auf die der Patient wissenschaftlich nachweisbar angewiesen ist, wird man aber nur sehr selten brauchen. Hier kann man dann durchaus, um das Leben zu retten, eine Überweisung vornehmen. Diese spezialistischen Indikationen sind in der Regel passager und brauchen nicht auf weite Sicht die Kontinuität der Betreuung. Gegebenenfalls müsste der Patient vielleicht in den zugehörigen Distrikt umziehen.

In der Mehrzahl der Fälle handelt es sich um stationäre Indikationen, die vor Ort erfüllt werden können und die auch eine kontinuierliche Betreuung vor Ort verlangen. Man denke an Leute mit einem Alkoholdelir, einer akuten schizophrenen Psychose oder einer schweren phasenhaft rezidivierenden manischdepressiven Erkrankung.

Bei dem zuletzt behandelten Thema des Krankenhauses handelt es sich beispielhaft um eine strukturelle Frage, die natürlich noch viel zwingender im Sinne des Sektors beantwortet werden muss, wenn es um den Aufgabenbereich der Tagesklinik oder der Institutsambulanz geht.

Im folgenden sollen die Heilmittel besprochen werden, die Pharmaka, die Psychotherapie und die sozialen Einflussnahmen.

Die Pharmaka kann man an jedem Ort geben, am Bodensee genauso wie auf dem Matterhorn. Von besonderen Applikationsformen abgesehen, z.B. über den Venenkatheter, spielt die Art der helfenden Einrichtung für die Medikamentenzufuhr keine Rolle.

Deutlich schwieriger werden die Verhältnisse bei der Psychotherapie. Natürlich kann man auch sie an jedem beliebigen Ort anwenden, zumal man dazu keine Injektionsnadeln und keine Schockgeräte braucht. Das Problem besteht nur darin, dass die Leute, die sie im täglichen Umgang anwenden sollen, nicht genügend Bescheid wissen - und dass die Leute, die sie beherrschen, sich oft am falschen Ort befinden, auf jeden Fall nur sehr selten innerhalb der "psychiatrie des pauvres». Bei aller Wertschätzung, die den einzelnen psychotherapeutischen Verfahren entgegenzubringen ist, scheint ggf. der Mangel an diesermassen spezialistischer Behandlung aber vertretbar zu sein. In der "psychiatrie des pauvres» haben Lebenserfahrung, Berufserfahrung, Sorge um die Familie und um den Arbeitsplatz vergleichsweise ein grösseres Gewicht. 
Anders ist es bei einer Gruppe, die jetzt häufiger zur Behandlung kommt, die erst kürzlich als "psychotherapeutische Medizin" und als "Psychosomatik» abgegrenzt wurde und für die auch ein eigener Facharzttitel entwickelt wurde. Hier muss man ehrlich zugeben, dass die klassischen Psychiater im allgemeinen nicht genügend Bescheid wissen. Falls es psychotherapeutische Techniken gibt, die dieser wachsenden Gruppe von Menschen mit Sicherheit helfen, dann müssen sie jederzeit und für jeden verfügbar angeboten werden. Man darf dabei aber nicht vergessen, dass auch diese Menschen normale Menschen sind, die nach dem Überstehen der akuten Störung eine kontinuierliche Betreuung - sowohl von der Person als auch von der Idee her - brauchen.

Man wird die hier gestellte Frage von Region zu Region neu beantworten müssen. Wenn eine grosse Bevölkerungsdichte besteht und die Wege nicht $\mathrm{zu}$ weit sind, kann sich z.B. einer der Sektoren, der im übrigen aber auch alle anderen Aufgaben wahrnehmen muss, darauf spezialisieren. In der Regel ist es allerdings erstrebenswert, dass die Bürgerinnen und Bürger innerhalb ihres Sektors alles vorfinden, was sie brauchen.

Das dritte noch zu nennende Heilmittel der Soziotherapie ist ziemlich eindeutig zu lokalisieren. Man kann einem Lebewesen am besten dort helfen, wo es zu Hause ist. Um mit Mosher zu sprechen: Man muss die Tiere nicht aus dem Dschungel in den Zoo bringen, damit man ihnen etwas Gutes tun kann, sondern man muss die Hilfe vor Ort entwickeln. [1]

Die örtlichen Lösungen haben alle den grossen Vorteil, dass man das kontrollieren und überwachen kann, was man veranlasst hat, dass es für den Benutzer zumutbar ist, im Kontakt zu bleiben, dass damit die Kontinuität des Behandlungskonzeptes und der persönlichen Beziehung gewährleistet ist, dass der soziale Platz durch die Benutzung der Hilfsangebote nicht ins Wanken kommt.

Vieles von dem, was als Erfordernis zu benennen war, wird deshalb nicht realisiert, weil man nicht an den anderen glaubt, weil man die banale Erkenntnis vergisst, dass Menschen es immer wieder lernen, von der Geburt bis zum Tod, mit den äusseren Bedingungen zurechtzukommen: greifen zu lernen, gehen $\mathrm{zu}$ lernen, eine Gefahr abzuwenden, ein Problem zu lösen, einen Krückstock zu benutzen usw. Die Fähigkeit, ein Problem lösen zu können, ist ein Wesensmerkmal des Lebendigen wie das Wachstum, die Reproduktionsfähigkeit und die Lokomotion.

Aber wie selten wird heute noch den Patienten unterstellt, dass sie ein Problem selbständig lösen können. Die ärztlichen Organisationsplanungen gehen immer davon aus, dass eine ganze Heerschar von Schutzengeln darauf aufpasst, dass alles gut geht. Das Analoge gilt für die Mitarbeiter anderer Berufsgruppen, z.B. des Pflegedienstes, die früher zu Befehlsempfängern degradiert worden waren. Seitdem aber z.B. in Merzig die Türen aufgemacht wurden und die Patienten nur dann nicht weggelaufen sind, wenn jeder einzelne Pfleger das von seiner Person einbrachte, was er am besten beherrschte und was ihn auszeichnete, zeigten diese Mitarbeiter ein neues und bisher nicht erwartetes Potential von Fähigkeiten [5]. Andererseits war bei den Patienten zu bemerken, dass sie darauf aufpassten, dass ein verwirrter älterer Herr nicht durch die Tür ging und die Station verliess, oder dass eine hinfällige alte Dame noch rechtzeitig aufgefangen wurde, bevor sie sich eine Schenkelhalsfraktur zuzog.

Und schliesslich fehlt es an Vertrauen in die persönlichen Fähigkeiten der Ärzte. So meint man, dass jeder Arzt nur in begrenztem Umfang Aufgaben meistern könne, dass es deshalb zentrale Lösungen geben müsse. Dabei wird ganz vergessen, dass jede Entlastung durch die Spezialisten oder durch die Zentrale auch einen Übungsverlust, eine Entkräftung bedeutet und dass man die Gelegenheit fortnimmt, das Gehen auf unwegsamem Gelände zu erlernen oder auf den Beinen zu bleiben.

Schliesslich meinen die Fachkrankenhäuser, dass bestimmte Aufgaben nur in ihren Mauern und mit ihrem Geist erfüllt werden können, so als ob sich diese Aufgaben nicht alle auch in kleinere Segmente verlagern liessen [6].

\section{Grenzen}

Zum Schluss soll noch auf den deutschen Begriff der Grenzen eingegangen werden.

In dem sektoriellen Zusammenhang hat das Wort Grenze mehrere Bedeutungen. Einmal geht es um die klare Linie, die anzeigt, wo die zugehörige Region jeweils aufhört. Dabei ist aufgrund psychiatriepolitischer Erfahrung zwingend zu verlangen, dass diese Linie in keinem Fall überschritten werden darf, schon deshalb, weil sie den Verantwortungsbereich der Verantwortlichen markiert. Solange man für sich selbst sorgt, wird ordentlich gearbeitet und gewirtschaftet. Sobald man etwas an andere delegiert, kann man seiner Sache nicht mehr so sicher sein. Landrat, Polizeipräsident, Amtsgerichtsdirektor, Amtsärztin und andere müssen wissen, was mit ihren Leuten und mit ihrem Geld geschieht, müssen kontrollieren und müssen immer wieder einwirken, wenn etwas falsch läuft oder noch nicht vorhanden ist. Wenn aus der Merziger Klinik ein Patient in eine Einrichtung verlegt wird, z.B. in ein Wohnheim, dann muss jedes Mal ein Zettel ausgefüllt und dem Chef zur Überprüfung vorgelegt werden. Auf ihm ist ausgeführt, warum dieser Mensch nicht in seiner Wohnung bleiben kann und wohin er nun kommen soll. Sobald die Grenzen des Landkreises mit 100000 Einwohnern überschritten sind, erfolgt keine Einwilligung.

Die Grenzen bilden also die Garantie für die konsequente Realisierung des Sektorgedankens: dass nämlich alle Sorgen vor Ort gelöst werden. Andere könnten natürlich sagen (und sie tun es auch), dass die Leute, die so denken, wie hier ausgeführt wurde, begrenzt oder «beschränkt" sind, dass sie sich selbst Barrieren auferlegen, die eine bessere Versorgung ihrer Bürger verhindern. 
Es fragt sich allerdings, wer sich mehr Schranken auferlegt - und vor allem, wer seinen Patienten mehr Grenzen zieht: derjenige, der sie zur Verwirklichung spezieller Therapiekonzepte an einzelnen Orten zusammenführt oder derjenige, der dem Patienten das Recht auf den eigenen Weg einräumt: an dem Ort, an dem er lebt und mit Hilfen an allen Stellen und in allen Stadien, die bei ihm persönlich als unterstützungsbedürftig erkannt werden.

\section{Der alltägliche Weg ist der richtige Weg}

Mit dem Prinzip des Sektorgedankens ist das Terrain abgesteckt. Den passenden Weg - den begleitenden, therapeutischen, rehabilitativen Weg muss jeder selber finden. Von Hanns Philipzen stammt der Hinweis auf einen Dialog des Zen-Buddhismus:

«Ein Schüler fragte seinen Lehrer: 'Was ist der richtige Weg?» Der Meister erwiderte: ‘Der alltägliche Weg ist der richtige Weg.)

Wiederum fragte der Schüler: ‘Kann man diesen Weg erlernen?

Der Meister sagte: ‘Je mehr Du lernst, desto weiter kommst Du vom Weg ab.,

Darauf fragte der Schüler: ‘Wenn man dem Weg nicht durch Lernen näherkommen kann, wie kann man ihn erkennen?,
Da sprach der Meister zu ihm: «Der Weg ist nichts Sichtbares und nichts Unsichtbares. Er ist nichts Erkennbares und auch nichts Unerkennbares. Such ihn nicht, lerne ihn nicht, nenne ihn nicht! Sei weit und offen wie der Himmel, und Du bist auf dem richtigen Weg.ım

\section{Literatur}

1 Werner W (Hrsg.). Auflösung ist machbar - vom Grosskrankenhaus zur Dezentralisierung. Bonn: Psychiatrie-Verlag; 1998.

2 Mosher LR, Burti L. Psychiatrie in der Gemeinde. Grundlagen und Praxis. Bonn: Psychiatrie-Verlag; 1992.

3 BMJFFG. Empfehlungen der Expertenkommission der Bundesregierung zur Reform der Versorgung im psychiatrischen und psychotherapeutisch-psychosomatischen Bereich. Bonn: Aktion Psychisch Kranke; 1988.

4 Enquête über die Lage der Psychiatrie in der BRD. Hier: Zwischenbericht der Sachverständigenkommission. BT-Drucksache 7/1124, 1973

5 Bernardi 0, Gerber HG, Krisor M, Philipzen H, Schmitt R, Schulte T, et al. (Hrsg.). Psychiatrie mit offenen Türen. Offene stationäre Psychiatrie in der Pflichtversorgung. Stuttgart, New York: Georg Thieme Verlag; 2000.

6 Auerbach et al. Perspektiven der Krankenhauspsychiatrie Positionspapier: Beilage zur Psychiatrischen Praxis 1/2000. 